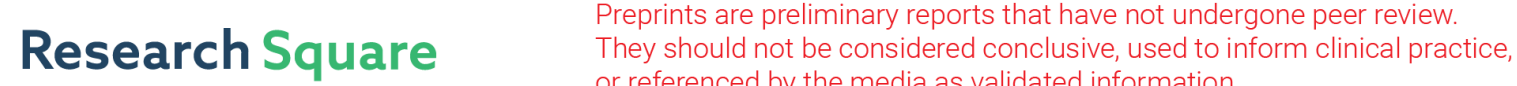 or referenced by the media as validated information. \\ Dynamic Picking and Storage Optimization of Robotic Picking Systems
}

\author{
Cheng Chi \\ Shandong University \\ Shasha Wu \\ Shandong University \\ Delong Xia \\ Shandong University \\ Yaohua Wu ( $\nabla$ yaohua.wu@sdu.edu.cn ) \\ Shandong University https://orcid.org/0000-0002-5561-8049
}

\section{Research Article}

Keywords: Robotic picking , Parts-to-picker , Storage allocation , Simulation analysis

Posted Date: July 9th, 2021

DOI: https://doi.org/10.21203/rs.3.rs-687207/v1

License: (c) (1) This work is licensed under a Creative Commons Attribution 4.0 International License. Read Full License 


\title{
Dynamic picking and storage optimization of Robotic Picking Systems
}

\author{
Cheng Chi ${ }^{1} \cdot$ Shasha Wu$^{1} \cdot \operatorname{Delong} \mathrm{Xia}^{1} \cdot$ Yaohua WU $^{1}$, *
}

\begin{abstract}
With the development of e-commerce and the improvement of logistics requirements, more and more 'parts-topicker' picking systems begin to replace the inefficient 'picker-toparts' picking systems in various scenarios. As the mainstream 'parts-to-picker' system, the robotic mobile fulfillment system has been attracting much attention in recent years. In addition to the customer's changing requirements, the rapid response of the picking system to the order is particularly important. In the above context, to seek a breakthrough in the picking system's picking efficiency without increasing the cost of additional equipment, the storage allocation of the pods becomes very important. This article focuses on the dynamic storage allocation of robotic mobile fulfillment system, which has positive theoretical and practical significance. By analyzing the pod storage process of the robotic mobile fulfillment system, a dynamic pod storage allocation model suitable for the robotic mobile fulfillment system is established with the goal of minimizing the pod handling distance. Two dynamic pod storage allocation strategies are proposed for the system. By simulating the picking systems of different scales, the effectiveness of the dynamic storage allocation strategy is verified, which has a certain reference to the operation of the robotic mobile fulfillment system in practice.
\end{abstract}

Keywords: Robotic picking $•$ Parts-to-picker $•$ Storage allocation - Simulation analysis

\section{Introduction}

With the continuous development of automatic and ecommerce technology in recent years, many kinds of mobile robots have been widely used and studied in modern intelligent warehousing field. Robotic Mobile Fulfillment System (RMFS) which is a storage and retrieval system that

1. College of Control Science and Engineering, Shandong University, Jinan 250061 China

*Correspondence: Yaohua WU, yaohua.wu@sdu.edu.cn uses autonomous robots to transport movable pods with items came into being widely used in e-commerce distribution centers ${ }^{[1]}$.

The concept of RMFS has been first proposed by Jünemann in $1989^{[2]}$. In 2008 , the most common RMFS system which names 'Kiva systems' applied a patent in the United States, which was later acquired by Amazon and renamed 'Amazon robot' ${ }^{[3]}$. Currently, the systems are running in several order fulfillment centers of Amazon around the world. RMFS has strong expansibility and flexibility that is because it can rapidly improve the throughput capacity of the system by increasing the number of robots and pickers during the peak period, such as shopping festival. Due to its flexibility, automation and intelligence, RMFS has been widely used in the field of practice. Since its launch,it has been successfully applied to many major e-commerce order fulfillment centers around the world, including Amazon, Walgreens, Staples, Jingdong, Alirookie, etc ${ }^{[4]}$.

The main operation process of RMFS includes not only order batching, task allocation to robots manual picking, pod automatic returning, but also the robot charging and pod replenishment. How to improve the efficiency of each process and the cooperation in processes, has related to the response speed of the entire warehouse center and the customer satisfaction with sales.

However, compared with the heat in the field of practice, there are few literatures on the theoretical research of RMFS. According to different research purposes, it can be divided into three categories: system analysis, design optimization and operation strategy.

Among them, the operation strategy belongs to the decision-making of short-term execution level, and its main 
goal is to shorten the delivery time, reduce the waiting time, improve the order response speed and reduce the equipment vacancy rate. This kind of decision-making mainly includes vehicle task allocation, congestion prevention mechanism, equipment stand-by strategy (that is, the selection of parking point after equipment completes a task), storage location allocation and work station task allocation. Nigam et al. (2014) ${ }^{[5]}$ studied the storage strategy of mobile pods. Compared with random storage strategy, closed open allocation of removable pod units results in low utilization of storage space. However, the closed open storage strategy can slightly improve the order throughput. Yuan et al. [6] studied the rate based reservoir allocation strategy of RMFS and constructed a continuous fluid model. Rate based storage allocation strategy means that according to the bestselling degree of goods, the more popular goods are stored closer to the picking platform. Compared with the random storage allocation strategy, a two or three class rate based storage allocation strategy can reduce the total moving distance of robot by $8 \%$ to $10 \%$. Lamballais et al. ${ }^{[7]}$ pointed out that using $\mathrm{ABC}$ classification based on product turnover rate, the maximum order throughput of the system can be increased by $50 \%$. Lamballais et al. assume that only one item is stored in a pod unit. Therefore, for multi-line order picking, multiple pod units are needed. However, in practice, a pod unit can store multiple items of goods. Therefore, a single pod unit may be able to meet the needs of a multi-line order. In order to solve this problem, Lamballais et al. further expanded their research, distributed products to multiple storage units, and built a SOQN model to estimate throughput time. Then, to minimize throughput time, the number of pod units allocated for each item, the ratio of picking station and replenishment station, and the replenishment level of each pod unit are optimized. The results show that in order to minimize the throughput time, the more pod units the inventory should be distributed, the better. In addition, they found that the best ratio of picking platform and replenishment platform is 1 to 2 , and the best replenishment level of pod unit is $50 \%$. Boysen et al. ${ }^{[8]}$ studied the order scheduling problem of picking stations in RMFS. They abstracted the problem as a mixed-integer programming problem. The results show that compared with the first come first service order scheduling strategy, the optimal order scheduling strategy can save half of the number of robots. They also proved that the number of robots can be further reduced by introducing the shared storage strategy (and the same item can be stored in multiple pod units). Zou et al. (2019) ${ }^{[9]}$ established a SOQN model based on the battery charging process to study the different battery power recovery strategies of RMFS and pointed out that the wireless induction charging strategy is the best. At the same time, the model realizes the efficiency estimation of battery replacement strategy and wired charging strategy.

This paper selects the pod storage location allocation direction as the research object, and optimizes the picking efficiency without increasing the original equipment cost. This research is of great value to improve the throughput and customer satisfaction of the picking center, and it is helpful to improve the speed of logistics in the process of picking. It is also a reference for the optimization of RMFS in various application scenarios.

\section{DESCRIPTION AND MODELLING}

\section{1 problem description}

The purpose of studying the dynamic pod storage problem of RMFS is to obtain the best matching between the storage position of the pod and the order picking demand. Therefore, the aim is to design the system dynamically adjusts the position of the pod in the storage area according to the real-time order so that the pod storage position of the picking system has certain adaptability to the requirement ${ }^{[10]}$.

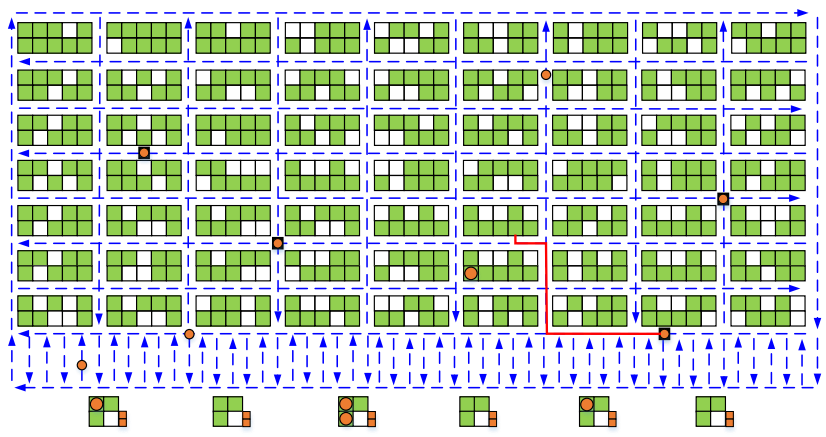

Figure 1 Dynamic storage allocation

In the picking process of the RMFS, the storage operation of the pod is one of the most frequent operations. Optimizing the storage position of the pod is an effective way to improve the efficiency of the system. ${ }^{[11]}$ During the order picking process, the robot moves the required pod from the storage area to the picking station. After the picker completes the item picking process, then, the robot moves the corresponding pod from the picking station to the storage area (as is shown in Figure 1 by red line ). In the process of the pod returning to warehouse, the system will reallocate the storage space of the pod according to certain optimization principles, so that the new storage position of the pod can meet the needs of order picking. With the continuous order picking process, the above process 
constantly optimizes the location of each pod, making the storage structure of the entire picking system match the demand for order picking.

The moving distance of the pods is closely related to the efficiency of the picking system. In this paper, the dynamic storage allocation model of the picking system is established to minimize the handling distance of the pods in the picking process. Different dynamic storage strategies are designed for the model. The simulation of the picking system is used to simulate the actual storage process of the pods, and different configuration systems are obtained under the optimal strategy.

\subsection{System Assumption}

To facilitate the analysis of the model without losing generality, the following assumptions are made for the model:

(1) the congestion, queuing or collision of the storage robot in the driving process is not considered;

(2) the goods in the pod are sufficient in the picking process, and the replenishment situation is not considered;

(3) the energy consumption problem is not considered, and the storage robot power is sufficient in the picking process;

(4) The task quantity of each picking station is balanced, and the whole picking process is periodic;

(5) the order combination processing is already done in the picking system so that a pod only serves one picking station in the same picking cycle, and multiple picking stations will not compete for one pod at the same time;

(6) The location coordinates of the whole storage area remain unchanged, and the shortest distance from the fixed location to the picking table is a fixed value.

Definition of parameters and variables is as the table bellow:

Table 1 Definition of parameters and variables

\begin{tabular}{|c|c|}
\hline Symbol & Meaning \\
\hline$R$ & $\begin{array}{l}\text { The total row number of the pods in the } \\
\text { warehouse }\end{array}$ \\
\hline C & $\begin{array}{l}\text { The total column number of the pods in the } \\
\text { warehouse }\end{array}$ \\
\hline$S$ & $\begin{array}{l}\text { Number of picking stations in the picking } \\
\text { system }\end{array}$ \\
\hline$P$ & Number of pods in the picking system \\
\hline$N$ & Number of AGV \\
\hline$H$ & Number of all SKUs in the picking system \\
\hline$T$ & The total period of the picking process \\
\hline$T^{\prime}$ & The set of picking period $\{1,2, \cdots, T\}$ \\
\hline$t$ & Picking period $t \in T^{\prime}$ \\
\hline
\end{tabular}

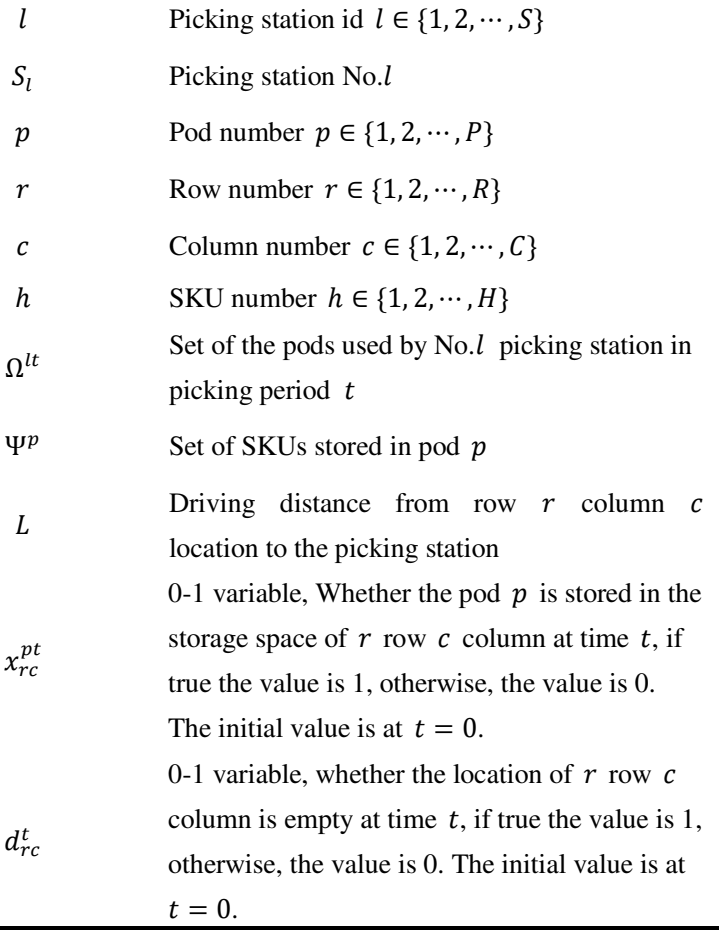

\subsection{System dynamic storage allocation model}

Under the above assumptions, the following dynamic storage allocation model is established according to the objective of minimizing the sum of pod moving distances in the picking process.

$$
\begin{gathered}
\min Z=\sum_{t=1}^{T} \sum_{l=1}^{S} \sum_{k \in \Omega^{l t}} \sum_{r=1}^{R} \sum_{c=1}^{C}\left(f_{A^{*}}\left(x_{r c}^{k(t-1)}, S_{l}\right)+\right. \\
\left.f_{A^{*}}\left(x_{r c}^{k t}, S_{l}\right)\right)
\end{gathered}
$$

s.t.

$$
\begin{gathered}
\sum_{r=1}^{R} \sum_{c=1}^{C} x_{r c}^{p t}=1 \quad \forall 1 \leq p \leq P, t \in T^{\prime} \\
\sum_{p^{\prime} \epsilon \Omega^{l t}} x_{r c}^{p^{\prime}(t-1)}+d_{r c}^{t-1}-\sum_{p \in \Omega^{l^{\prime}} t} x_{r c}^{p t}-d_{r c}^{t}=0 \\
r \leq R, 1 \leq c \leq C, 1 \leq l \leq S, 1 \leq l^{\prime} \leq S \\
\sum_{p=1}^{P} x_{r c}^{p t}+d_{r c}^{t}=1 \quad \forall 1 \leq r \leq R, 1 \leq c \leq C, t \in \\
f_{A^{*}}\left(x_{r c}^{k t}, S_{l}\right)= \begin{cases}L, & x_{r c}^{k t}=1 \\
0, & x_{r c}^{k t}=0\end{cases} \\
x_{r c}^{k t} \in\{0,1\} \\
d_{r c}^{t} \in\{0,1\}
\end{gathered}
$$

Where formula (1) is the objective function, which represents the sum of the moving distances of the pods in all periods in the whole picking process. Formula (2) indicates that at any time $t$, each pod has storage space. Formula (3) represents the relationship between the change of storage space in a continuous cycle. If the location of column $c$ 
row $r$ is empty or storing the pod used in time $t$ at time $t$, then the location is empty or storing the pod used in time $t$ at time $t-1$. Formula (4) indicates that at any picking time, the location of column $c$ row $r$ is empty or storing pod. Formula (5) calculates the moving distance of the pod. When $x_{r c}^{k t}=1$, i.e. the location of column $c$ row $r$ is storing the pod $k$, then the value is the moving distance of A* algorithm from column $c$ row $r$ to the picking station, otherwise, the value is 0 . Formulas (6) and (7) are constraints on variable values.

\section{DYNAMIC STORAGE STRATEGIES}

Because the problem is difficult to get the exact solution in a short time when the number of orders is large, it is necessary to design the heuristic strategy to simulate the whole model. In this problem, the position $x_{r c}^{k t}$ where the pod back to is the most important decision variable. Two kinds of dynamic storage strategies are designed based on the storage location of the pod: partition dynamic storage strategy and buffer-based storage strategy. ${ }^{[12]}$

\subsection{Partition dynamic storage strategy}

\subsubsection{Region dividing}

Region dividing is the first step in the implementation process of partition dynamic storage strategy: Area A for best-selling products, area $\mathrm{B}$ for general sales, and area $\mathrm{C}$ for unsalable products. The main purpose of the regional division is to allocate the cargo space reasonably according to the demand frequency of SKU. Due to different standards, the regional division can be divided into distance strategy and time strategy.

According to the distance partition strategy (as shown in Figure 2(a)), as a classic ABC partition strategy, it is widely used in the traditional mannalwarehouse. The warehouse area is divided according to the average distance from the storage area point to the picking platform. Euclidean and Manhattan are two commonly used distance selection methods ${ }^{[13]}$.

\begin{tabular}{|c|c|c|c|c|c|} 
Area \\
Area
\end{tabular} A

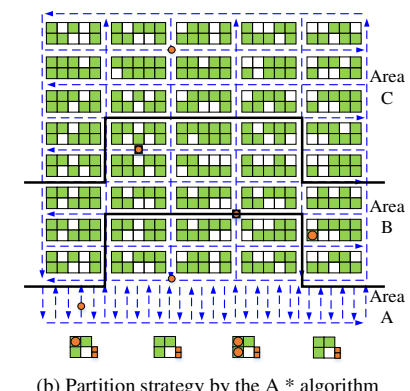

Figure 2 Partition of the storage area

The time partition strategy uses the A * algorithm ${ }^{[14]}$ with the shortest walking time to partition the whole storage area. In the whole process of partition, the shortest time from each storage area point to the picking platform is calculated according to the $\mathrm{A} *$ algorithm (including the time of robot turning) and then sorted according to the length of time. Then, according to the proportion of different areas, each storage area point is assigned the area type. The shortest time is assigned to area A, and the longest time is assigned to area $\mathrm{C}$. Compared with the distance partition strategy, the time partition strategy integrates some characteristics of the pod handling robot picking system, such as one-way driving in the roadway, and considers various physical movement characteristics of the handling robot, so that the best-selling area, unsalable area and other areas after partition are more suitable for the picking system.

Through the simulation of the time partition of the pod handling robot picking system, the partition (as shown in Figure 3 ) is roughly consistent with the description of the schematic diagram in Figure 3-2 (b), in which the regional proportion is generated according to $3: 3: 4$, and the regional distribution of most of the best-selling areas and unsalable areas fit the schematic diagram. However, the regional distribution is not completely regular, and a small number of reservoirs are distributed across regions. The reason for this phenomenon is that in the pod handling robot picking system, to improve the robot handling efficiency, the storage area roadway is a single lane, and some of the storage spaces are too many turns in the process of walking from the picking platform to the storage area, resulting in a long walking time. As the standard of regional division is planned according to the driving time, the distribution of a small part of the area is irregular.

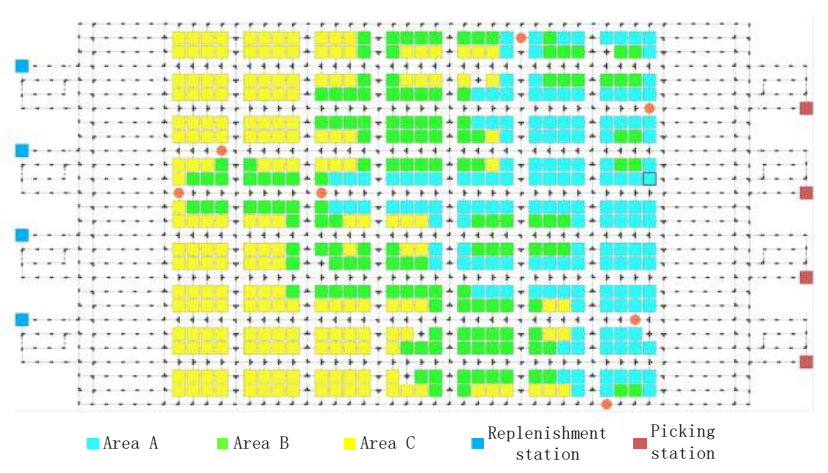

Figure 3 Schematic diagram of time partition in simulation

\subsubsection{Partition dynamic storage strategy based on pod}




\section{turnover rate}

In the partition dynamic storage strategy based on pod turnover rate, pod turnover rate becomes an important basis for dynamic storage allocation. The calculation method of the pod turnover rate is the cumulative number of times the pod is used. After each picking, the turnover rate of all pods will be updated in real-time, to ensure the reliability of data in decision-making.

In equation $(8), p(p, l, t)$ indicates whether the pod $p$ is used by the picking table $l$ in the $t$ cycle. If it is, the value is 1 ; otherwise, the value is $0 . f_{1}\left(p \cdot t^{\prime}\right)$ is the turnover rate of the pod $p$ at time $t^{\prime}$, which is calculated as shown in formula (9) by accumulating the number of times pod $p$ used in the historical period.

$$
\begin{aligned}
& p(p, l, t)=\left\{\begin{array}{l}
1, p \in \Omega^{l t} \\
0, p \notin \Omega^{l t}
\end{array}\right. \\
& f_{1}\left(p \cdot t^{\prime}\right)=\sum_{t=1}^{t^{\prime}} \sum_{l=1}^{S} p(p, l, t)
\end{aligned}
$$

According to this strategy, when calculating the location of pod $p$, the storage location of the pod is determined by obtaining the ranking of the circulation rate $f_{1}\left(p . t^{\prime}\right)$ of pod $p$ among all pods. If the turnover rate $f_{1}\left(p . t^{\prime}\right)$ of the pod $p$, is less than the capacity of the bestselling area in all the pods, and there is still space in the bestselling area, the pod is allocated to space nearest to the picking table in the best-selling area. If the turnover rate 1 $f_{1}\left(p \cdot t^{\prime}\right)$ of $p$ is greater than the sum of the capacity of the best-selling area and the general storage area, the pod will be allocated to the unsalable area; otherwise, the location will be allocated to the general sales area $\mathrm{B}$.

\subsubsection{Partition dynamic storage strategy based on the item flow rate}

Similar to the strategy based on the turnover rate of pods, the partition strategy based on item turnover rate pays more attention to the turnover rate of each item in the pod. After each picking at the picking station, the system will automatically increase the flow rate of the items involved in the picking. Because one item exists in multiple pods, the utilization rate of all related pods will increase, thus providing reliable data support for decision-making.

In equation (10), $q(h, p)$ indicates whether the goods stored in pod $p$ contain item $h$. If so, the value is 1 ; otherwise, the value is 0 . In equation (11), $d_{t^{\prime} h}$ is the cumulative times of picking item $h$ at the time $t^{\prime}$. In (12), $f_{2}\left(p, t^{\prime}\right)$ means the comprehensive turnover rate of pod $p$ at the time $t^{\prime}$ is calculated according to the item turnover rate.

$$
\begin{gathered}
q(h, p)=\left\{\begin{array}{l}
1, h \in \Omega^{p} \\
0, h \notin \Omega^{p}
\end{array}\right. \\
d_{t^{\prime} h}=\sum_{t=1}^{t^{\prime}} \sum_{l=1}^{S} p(p, l, t) q(h, p) \\
f_{2}\left(p, t^{\prime}\right)=\sum_{h \in \Omega^{p}} d_{t^{\prime} h}
\end{gathered}
$$

When calculating the storage location of pod $p$, the system will integrate the circulation rate of all items in the pod to obtain the ranking of $f_{2}\left(p, t^{\prime}\right)$ corresponding to pod $p$ in all pods, and allocate the corresponding storage area and storage location according to the ranking of the pod. If the pod is in the top rank to meet the requirements of the best-selling area $\mathrm{A}$, and there are free storage spaces in this area, the storage space nearest to the picking station is selected as the storage point. Otherwise, the storage space is selected in the general area and the unsalable area according to the ranking of each item flow rate of the pod.

\subsection{Buffer-area based dynamic storage strategy}

\subsubsection{Buffer partition}

The storage strategy based on buffer area is to divide a fast buffer area in the whole storage area and store the recently used pod in this area temporarily.Therefore, when the pod is needed for reordering,it can effectively reduce the handling distance of the robot, so as to reduce the storage time of the pod, improve the throughput of the picking station, and increase the transportation efficiency of the whole system efficiency.

The partition standard of buffer area is consistent with the time algorithm in 3.1 , and the $\mathrm{A}$ * shortest time algorithm is adopted. We sort the average time from all storage points to the picking station and select the storage area with the shortest time as the buffer area to divide the whole storage area into two parts: the buffer area and the conventional storage area (as shown in Figure 4).

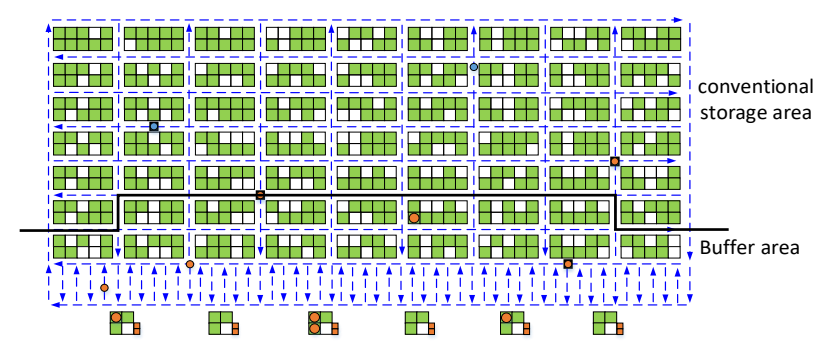

Figure 4 Diagram of the buffer storage layout

Because the pods that have just been used will enter the buffer area for temporary storage, if some pods in the buffer 
area are not eliminated in time, the entire buffer area will be piled up too much or even reach the upper limit, which will cause serious robot congestion in the buffer area, and then affect the operation efficiency of the buffer area. To avoid the backlog in the buffer area, when the number of pods stored in the buffer area reaches a certain threshold, the pods in the buffer area will be eliminated according to a certain strategy, and the handling robot (blue robot shown in Figure 4-2) will carry the pods to the conventional picking area. According to the different elimination modes, the bufferarea based dynamic storage strategy can be divided into four types: first in first out (FIFO) elimination mode, the elimination mode according to the frequency, the least frequently used elimination mode and the random elimination mode.

\subsection{2 first in first out elimination mode}

FIFO elimination mode is a strategy to eliminate the pods in the buffer area of the picking system according to the order of arrival. $\Omega_{p}^{t}$ is used to represent the pods set of the buffer at time $t$. By comparing the time of pod entering buffer area $t(p), p \in \Omega_{p}^{t}$, the corresponding pods $p^{\prime}$ satisfying $\min t\left(p^{\prime}\right), p^{\prime} \in \Omega_{p}^{t}$ is selected as the pods eliminated in the buffer area.

In the pre-processing operation of the system's picking work, such operations as order batching and order fulfillment sequence optimization make the recently used pods be used in the subsequent picking probability. Due to the limitation of buffer capacity in the system with FIFO strategy, if the storage capacity of the pods in the buffer reaches a certain threshold, the mobile robot will move the pod that first enters the buffer to the nearest free conventional storage area. While ensuring that the buffer can effectively store the nearest pod, the mobile robot will eliminate the pod that first enters the buffer. This strategy can greatly improve the efficiency of the system when cooperating with the order clustering operation.

\subsubsection{Elimination mode according to the frequency of use}

According to the use frequency elimination mode is a strategy to eliminate the pods in the buffer area of the picking system according to the use frequency of the pods. During the picking process, the system will record the use frequency of each pod in real-time. When the storage capacity of the pods in the buffer reaches a certain threshold, the mobile robot will move the pods with the lowest frequency in the buffer to the nearest conventional storage area, so that the pods stored in the buffer are the pods with higher frequency.

Before eliminated pod $p^{\prime}$ is selected, the utilization frequency $f_{1}\left(p^{\prime}, t^{\prime}\right), p^{\prime} \in \Omega_{p}^{t}$ of all pods in the buffer area is calculated first. Traversing the pods in $\Omega_{p}^{t}$, we find the pods $p^{\prime}$ with the least use frequency, such that $f_{1}\left(p^{\prime}, t^{\prime}\right)=$ $f_{1}\left(p, t^{\prime}\right), p \in \Omega_{p}^{t}$. Finally, pod $p^{\prime}$ is selected as the pod to move out of the buffer.

Because the change of turnover rate is closely related to the order structure, the efficiency of the system under this strategy is greatly affected by the order structure. If the order structure is unstable, the update speed of the buffer area will be faster, which will reduce the capacity of the buffer and increase the walking time of the robot. Finally, the throughput of the picking station will be reduced, and the expected work efficiency can not be achieved.

\subsubsection{The least frequently used elimination mode}

The most infrequently used elimination mode is to eliminate the pods in the buffer area of the picking system according to the latest usage time of the pods. The least frequently used elimination strategy is one of the commonly used page replacement algorithms in computer memory paging. It is used to maximize the probability of hitting the cache page and reduce the interruption of the operating system due to page missing. It is also a common algorithm for various large websites to deal with the back-end cache.

The pods in the buffer area are regarded as a queue when they are carried by the handling robots in the system. Whenever the pod is moved from the picking station to the buffer area in period $t$, or whenever the new pod enters the buffer storage area, the pod is placed at the head of the queue. Once the queue capacity reaches the threshold, the pod at the end of the queue will be eliminated directly.

\subsection{3 random elimination mode}

The random elimination strategy is to eliminate the pods in the buffer area of the picking system at random. By introducing random quantity, when the number of pods stored in the buffer reaches the specified threshold, the pods that are not pre-selected in the buffer are randomly selected and moved to the nearby conventional storage area.

For random selection, the system randomly generates the pod number $p^{\prime}=\operatorname{random}\left(\Omega_{p}^{t}\right)$, and moves the pod number $p^{\prime}$ out of the buffer.

In the process of random selection, the efficiency of system operation is unstable due to the influence of probability. Under this strategy, the operation efficiency is less affected by the order structure, and the influence of random quantity becomes the primary factor. 


\section{RESULTS ANALYSIS}

\subsection{Simulation design}

This simulation uses software RAWSim- ${ }^{[15]}$ as the overall framework to independently develop the above strategies in the controller layer. RAWSim-O was developed by Merschformann and his parterners. As a simulation software for RMFS, RAWSim-O has many advantages, such as open source, easy to use, visualization and so on. It has become a high-quality RMFS simulation software after AlphabetSoup.

The architecture of RAWSim-O is shown in Figure 5. The software provides various entities, complete underlying code and some basic strategy interfaces. Moreover, the software is an open source software. Researchers can carry out secondary development according to their own strategies and reuse the underlying framework code, thus greatly reducing the repeated workload of researchers.

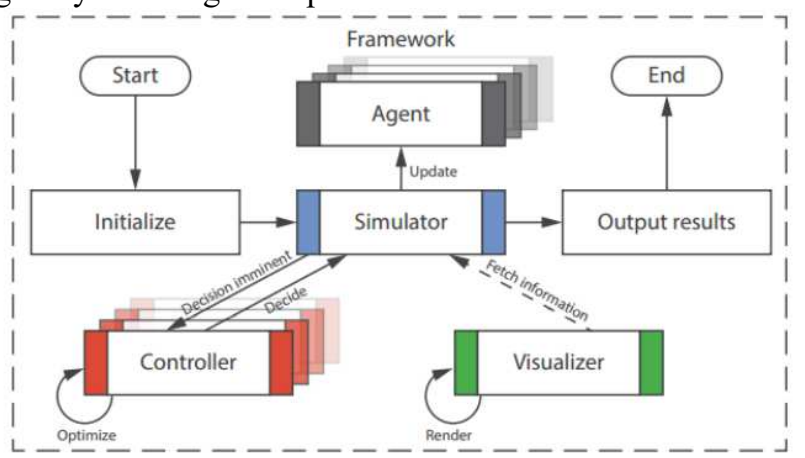

Figure 5 Software architecture diagram of the RAWSim-O

In order to simulate the warehouse picking situation under different scales and increase the reliability of the strategy, this simulation designs three warehouses of different scales: small-scale warehouse, medium-scale warehouse and large-scale warehouse, and subdivides the warehouses under three different scenarios under different scales, with a total of nine different warehouse scales. Independent simulation experiments are carried out for nine scenarios to explore the effects of various storage strategies on the pod handling distance and operation efficiency in different scenarios.

Table 2 shows the basic parameters under different warehouse scenarios, where $R$ is the total row number of pods in the direction of the picking station, $C$ is the total column number of pods in the storage area, $P$ is the total number of pods in the storage area, $S$ is the number of picking stations in the system, $N_{h}$ is the number of lanes in the horizontal direction of the storage area of the picking system, $N_{v}$ is the number of lanes in the vertical direction,
$N_{b}$ is the total number of handling robots in the picking system, including the number of eliminated robots in the buffer storage strategy, so as to ensure the consistency of picking conditions under different strategies.

Table 2

\begin{tabular}{|c|c|c|c|c|c|c|c|c|}
\hline $\begin{array}{c}\text { Warehous } \\
\text { e size }\end{array}$ & Scenario & Row & Column & Pod & Station & $N_{h}$ & $N_{v}$ & $N_{b}$ \\
\hline \multirow{3}{*}{$\begin{array}{l}\text { small- } \\
\text { scale }\end{array}$} & $\begin{array}{c}\text { Scenario } \\
1\end{array}$ & 25 & 6 & 102 & 1 & 2 & 4 & 3 \\
\hline & $\begin{array}{c}\text { Scenario } \\
2\end{array}$ & 25 & 10 & 170 & 2 & 4 & 4 & 6 \\
\hline & $\begin{array}{c}\text { Scenario } \\
3\end{array}$ & 25 & 14 & 238 & 3 & 6 & 4 & 9 \\
\hline \multirow{3}{*}{$\begin{array}{l}\text { medium- } \\
\text { scale }\end{array}$} & $\begin{array}{c}\text { Scenario } \\
4\end{array}$ & 35 & 14 & 333 & 3 & 6 & 6 & 9 \\
\hline & $\begin{array}{c}\text { Scenario } \\
5\end{array}$ & 35 & 18 & 428 & 4 & 8 & 6 & 16 \\
\hline & $\begin{array}{c}\text { Scenario } \\
6\end{array}$ & 45 & 18 & 550 & 4 & 8 & 8 & 16 \\
\hline \multirow{3}{*}{$\begin{array}{l}\text { large- } \\
\text { scale }\end{array}$} & $\begin{array}{c}\text { Scenario } \\
7\end{array}$ & 45 & 22 & 673 & 5 & 10 & 8 & 25 \\
\hline & $\begin{array}{c}\text { Scenario } \\
8\end{array}$ & 55 & 22 & 822 & 5 & 10 & 10 & 30 \\
\hline & $\begin{array}{c}\text { Scenario } \\
9\end{array}$ & 55 & 26 & 972 & 5 & 12 & 10 & 30 \\
\hline
\end{tabular}

Figure 6 is the storage layout diagram of Scene 3, Scene 5 and Scene 7 in the above storage layout. According to the above information of roadway, pod and picking station, the layout of real storage is simulated to restore the actual picking process to the greatest extent, and the direction of each roadway and the path of the picking station are planned, to ensure the safety and efficiency of the handling robot in the process of driving, and provides a guarantee for the reliability of simulation data. 


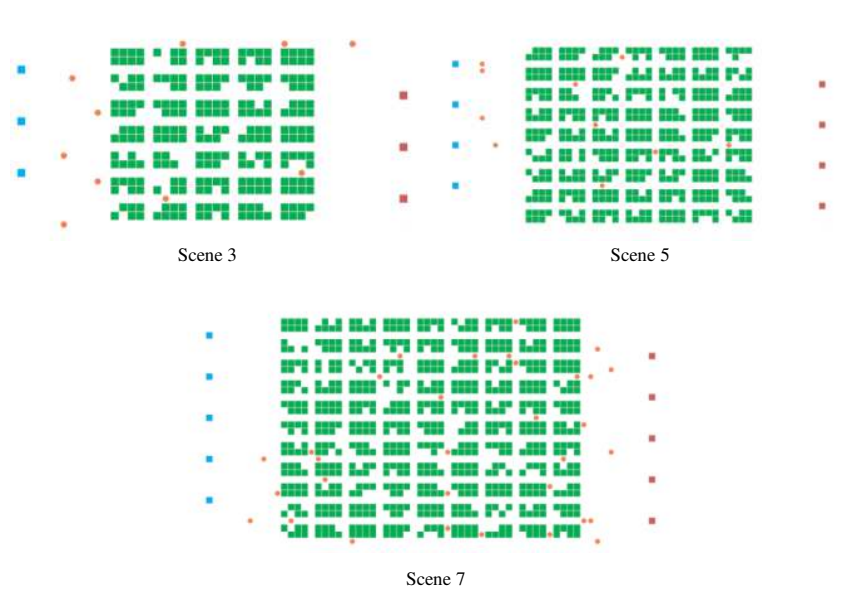

Figure 6 Simulation diagram of partial scale warehouse layout

Table 3 describes the properties of some pod robots, the properties of pods and other conventional parameters. This part of the parameters is obtained by referring to the part of the warehouse data to simulate the picking situation in the real warehouse as far as possible. Based on the following parameters, we restore a variety of actual scenes, such as the robot carrying pods, one-way driving in the roadway, picking platform picking and so on in the simulation, in order to analyze different operations

Table 3 General parameter of the picking system

\begin{tabular}{l|c}
\hline Parameter & Value \\
\hline $\begin{array}{l}\text { The absolute value of maximum } \\
\text { acceleration/deceleration of the mobile robot } a\end{array}$ & $1 \mathrm{~m} / \mathrm{s}^{2}$ \\
$\begin{array}{l}\text { The maximum speed of the mobile robot } v \\
\text { The time required for the mobile robot to rotate } 90 \\
\text { degrees } t_{1}\end{array}$ & $2 \mathrm{~s} / \mathrm{s}$ \\
$\begin{array}{l}\text { The time required for the mobile robot to lift/put } \\
\text { down pods } t_{2}\end{array}$ & $2.2 \mathrm{~s}$ \\
$\begin{array}{l}\text { The maximum quantity of SKUs that can be stored on } \\
\text { a single pod } N_{p}^{c}\end{array}$ & 500 \\
$\begin{array}{l}\text { The total time for a picker to pick an SKU and put it } \\
\text { in the picking box }\end{array}$ & $3 \mathrm{~s}$ \\
\hline
\end{tabular}

\subsection{Results}

The simulation results are divided into three parts: partition dynamic storage strategy results, dynamic storage strategy based on buffer area results and Comprehensive comparison of strategies. The results of partition dynamic storage strategy and dynamic storage strategy based on buffer area are mainly the effects of different partition or buffer elimination schemes. The part of strategy comprehensive comparison is to explore the advantages and disadvantages of each strategy and provide better solutions for different application scenarios.

\subsubsection{Analysis of partition dynamic storage strategy simulation results}

Firstly, two different regional classification standards are compared to determine the impact of different classification standards on the test as a whole. In order to ensure the comprehensiveness of the application scenarios in the simulation, experiments are carried out for the different number of handling robots and different warehouse sizes, so as to comprehensively compare the impact of two different classification standards on the handling robot picking system. In the simulation, the system is studied under the three determined partition ratios, that ratios are fixed as $\theta_{a}=0.2, \theta_{b}=0.3$ and $\theta_{c}=0.5$. And the turnover rate adopts the operation situation under the item turnover rate calculation mode. One scene is selected for simulation in each of the three different storage scales, so as to get the sum of the distance between the picking station and the storage area of the handling robot of the two area division standard systems in different scenes. The simulation results of the two partition methods in scenario 3 , scenario 5 and scenario 7 are shown in table 4 .

Table 4 shows the comparison of the two partition strategies in different scenarios, in which the value represented by the data is the carrying distance between the picking station and the storage area (unit: $\mathrm{m}$ ) during the picking process. It can be seen from the data in the table that in three different scenarios, the carrying distance of the system under the time partition strategy is smaller than that under the distance partition strategy. The main reason for this difference is that the roadways of the pod handling robot picking system are all unidirectional, and the simple distance as the partition consideration can not fully fit the walking path of the robot in the system, and the time strategy is more suitable for the system in the application scenario, so the picking distance is less than the system under the distance partition.

Table 4 Simulation comparison of two partition modes

\begin{tabular}{cccc}
\hline \multirow{2}{*}{$\begin{array}{c}\text { Warehouse } \\
\text { size }\end{array}$} & $\begin{array}{c}\text { Order } \\
\text { quantity }\end{array}$ & $\begin{array}{c}\text { Tod handling distance/m } \\
\text { strategy }\end{array}$ & $\begin{array}{c}\text { Partition } \\
\text { strategy }\end{array}$ \\
\hline Scenario 3 & 500 & 7540 & 7677 \\
& 1500 & 24735 & 25706 \\
& 2500 & 42651 & 45264 \\
\hline Scenario 5 & 500 & 7624 & 7681
\end{tabular}




\begin{tabular}{llll}
1500 & 25507 & 26311 \\
& 2500 & 45813 & 47357 \\
\hline Scenario 7 & 1500 & 8645 & 8735 \\
& 2500 & 28762 & 29483 \\
& 49612 & 50551 \\
\hline
\end{tabular}

Under the above conditions, we explore the influence of item turnover rate and pod turnover rate on the picking distance in the system picking process. In order to ensure the uniqueness of the variables in the simulation, the proportion of the storage area of the three partitions, $\theta_{a}=$ $0.2, \theta_{b}=0.3$, and $\theta_{c}=0.5$ is fixed, and then the system operation using the time partition mode is explored. One scene is selected for simulation in each of the three different scales of storage, so as to get the sum of the robot walking distance between the picking station and the storage area under two different flow rate calculation methods in different scenes. The simulation results of the two partition methods in scenario 3, scenario 5 and scenario 7 are shown in table 5 .

The data in table 5 shows the comparison of the two calculation methods of turnover rate under different scenarios, in which the value represented by the data is the carrying distance between the picking station and the storage area (unit: $\mathrm{m}$ ) during the picking process. By comparing the data of the two turnover rate calculation methods in the table we know that the picking and handling distance of the system based on the pod turnover rate calculation method is smaller than that of the system based on the item turnover rate calculation method. In Scene 3, Scene 5 and Scene 7, the average picking distance of the system based on the pod turnover rate is 137 meters, 401 meters and 505 meters shorter than the system based on the item turnover rate, respectively. The corresponding difference also gradually increases with the gradual increase of the storage scale. The location of each return is determined by the frequency of the pod in the pod turnover rate mode, while that location is determined by the average turnover rate of various items stored on the pod in the item turnover rate mode. Due to the situation that one kind of item can be stored in multiple pods, and the storage capacity of the best-selling area and the storage area with general sales volume is limited, the pod containing the same bestselling item may be repeatedly stored in the best-selling area under the calculation method of item turnover rate. But the calculation method based on the pod turnover rate will reduce the occurrence of this kind of situation. Therefore, the picking distance of the pod turnover rate calculation method in different scenarios is smaller than that of the picking system based on the item turnover rate.

Table 5 Simulation comparison of two calculation methods of turnover rate

\begin{tabular}{cccc}
\hline \multirow{2}{*}{$\begin{array}{c}\text { Warehouse } \\
\text { size }\end{array}$} & $\begin{array}{c}\text { Order } \\
\text { quantity }\end{array}$ & $\begin{array}{c}\text { Pased on item } \\
\text { turnover rate }\end{array}$ & $\begin{array}{c}\text { Based on pod } \\
\text { turnover rate }\end{array}$ \\
\cline { 3 - 4 } Scenario 3 & 500 & 7540 & 7509 \\
& 1500 & 24735 & 24471 \\
Scenario 5 & 2500 & 42651 & 42534 \\
\hline & 500 & 7624 & 7451 \\
& 1500 & 25507 & 25342 \\
\hline \multirow{2}{*}{ Scenario 7 } & 1500 & 45813 & 44920 \\
\hline & 2500 & 8645 & 8294 \\
& 500 & 28762 & 27920 \\
& 2500 & 49612 & 49289 \\
\hline
\end{tabular}

Through the above data analysis, we can know that in the above three scenarios, the time partition mode is more suitable for the picking system, and the partition storage strategy based on pod turnover rate is better than the partition storage strategy based on item turnover rate in all aspects.

\subsubsection{Analysis of buffer area dynamic storage strategy} simulation results

For the simulation experiment of buffer strategy, this paper mainly explores the influence of the proportion of buffer area in the total storage area $\theta_{b f}$, the elimination threshold of the buffer area $\varphi_{b f}$ and four elimination mode strategies of different buffer areas to the pod handling distance of the picking system, so as to find the suitable configuration for the picking system in different scenarios. Because the number of robots determined in the scenario is fixed, the buffer elimination does not use additional robots to ensure the consistency of the variable. The number of buffer elimination robots used in small-scale warehouse, medium-scale warehouse and large-scale warehouse is 1,2 and 3 respectively, and the rest robots are all used for picking tasks.

First of all, the size of the buffer $\theta_{b f}$ is explored. This paper takes $\theta_{b f}$ as the independent variable to explore the influence of different buffer sizes on the picking distance in 
the process of picking operation, so as to determine the optimal buffer proportion for different sizes of picking system. The simulation strategy adopts the FIFO strategy, and the buffer elimination threshold $\varphi_{b f}$ is 0.8 . In the case of the above parameters, the simulation results of the system in scenario 3, scenario 5 and scenario 7 are shown in table 5, in which $S 1, S 2, S 3, S 4$ and $S 5$ represent the picking system when $\theta_{b f}$ values are $0.1,0.15,0.2,0.25$ and 0.3 respectively.

It can be seen from the data in table 6 that the proportion of the buffer area in the picking area has an impact on the carrying distance of the picking pod. When $\theta_{b f}=0.15$, the picking distance is the shortest in most scenarios. It can be concluded that when the size of the buffer area accounts for about $15 \%$ of the whole storage area, the buffer area can better realize the buffer function of the pod used in the near future, and cooperate with the handling robot to play a better work efficiency and improve the throughput of the picking station. When the buffer area is small, the buffer is prone to accumulate, that is, the speed of entering the buffer area is faster than the speed of eliminating the buffer, which makes the buffer lose its due performance. When the buffer area is too large, the strategy of the whole buffer will be changed to the nearby storage strategy, and the elimination working distance of the buffer will be longer, thus reducing the overall efficiency of the system.

Table 6 Simulation comparison of different buffer sizes

\begin{tabular}{ccccccc}
\hline \multirow{2}{*}{$\begin{array}{c}\text { Warehouse } \\
\text { size }\end{array}$} & $\begin{array}{c}\text { Order } \\
\text { quantity }\end{array}$ & \multicolumn{5}{c}{ Pod handling distance/m } \\
\cline { 3 - 7 } & 500 & 6940 & 6700 & 6850 & 7166 & 7275 \\
\hline \multirow{2}{*}{ Scenario 3 } & 1500 & 23225 & 23140 & 24671 & 24156 & 23572 \\
& 2500 & 40461 & 40434 & 41009 & 41143 & 41219 \\
\hline & 500 & 7684 & 6915 & 7203 & 7590 & 7385 \\
& 1500 & 24953 & 25009 & 25843 & 25335 & 25911 \\
& 2500 & 44915 & 43338 & 45455 & 44859 & 46336 \\
\hline Scenario 5 & 500 & 8087 & 7518 & 7894 & 7766 & 7917 \\
& 1500 & 26039 & 24562 & 24873 & 25650 & 25153 \\
\hline & 2500 & 43909 & 42583 & 43094 & 43280 & 43442 \\
\hline
\end{tabular}

Table 7 shows the system performance comparison under different buffer elimination strategies, in which the data is the handling distance (unit: $\mathrm{m}$ ) between the picking platform and the storage area during the picking process.
Among them, strategy 1, strategy 2, strategy 3 and strategy 4 respectively represent the buffer dynamic storage strategies under the first in first out elimination mode, according to the frequency of use elimination mode, the least frequently used elimination mode and random elimination mode. Through the data in the table, the system of four elimination strategies is compared. Scenario 3 shows that in a small-scale warehouse, strategy 3 is better than strategy 2 when the order quantity is small. With the increase of order quantity, strategy 2 becomes the strategy with the least pod handling distance in the system picking process. Strategy 4 is a random elimination strategy with the worst performance. Scenario 5 is a storage scenario with a medium storage scale. In scenario 5 , the sequence of the four strategies is: strategy $2>$ strategy $3>$ strategy $1>$ strategy 4. Strategy 2 is to eliminate the pod in the buffer area according to the pod usage frequency, so as to clean the buffer area under the condition that the buffer area does not reach the upper limit, and improve the overall performance. Scenario 7 is a large-scale warehousing scenario. In this scenario, the order of the four strategies is strategy $2>$ strategy $1>$ strategy $3>$ strategy 4 . In the three scenarios, strategy 4 has the worst comprehensive performance among the four strategies, and strategy 2 has the best comprehensive performance.

Table 7 Simulation comparison of different elimination strategies

\begin{tabular}{cccccc}
\multirow{2}{*}{$\begin{array}{c}\text { Warehouse } \\
\text { size }\end{array}$} & Order & \multicolumn{5}{c}{ Pod handling distance/m } \\
\cline { 3 - 6 } & quantity & Strategy & Strategy & Strategy & Strategy \\
& 500 & 6700 & 6817 & 6611 & 7382 \\
\hline \multirow{2}{*}{ Scenario 3 } & 1500 & 23140 & 23066 & 23427 & 25088 \\
& 2500 & 40434 & 39758 & 39989 & 43461 \\
\hline \multirow{2}{*}{ Scenario 5 } & 1500 & 25009 & 22289 & 22662 & 26546 \\
& 2500 & 43338 & 40119 & 40624 & 44635 \\
\hline & 500 & 7518 & 7472 & 7627 & 7930 \\
& 1500 & 24562 & 23686 & 24351 & 27615 \\
\hline & & 6512 & 6646 & 7233 \\
& 2500 & 42583 & 41972 & 43874 & 48665 \\
\hline
\end{tabular}

\subsubsection{Comprehensive comparative analysis}

Through the above analysis of the system pod handling distance under the partition storage strategy and the buffer storage strategy, the optimal configuration of different 
strategies in different scenarios is obtained. The simulation comparison of various strategies under the above configuration is carried out, and the applicability of various strategies is analyzed by comparing the system throughput under various strategies and different warehouse sizes.

Among them, strategy $\mathrm{A}$ is the partition picking strategy, the dynamic partition is used in the partition, and the storage allocation is based on the pod turnover rate. Strategy B is the buffer dynamic storage strategy based on the usage frequency elimination mode, and the proportion of buffer area in the total storage area is $15 \%$. Among them, the number of buffer eliminated robots in Scene 1, 2 and 3 is 1 , the number of buffer eliminated robots in Scene 4, 5 and 6 is 2 , and the number of buffer eliminated robots in Scene 7, 8 and 9 is 3. Strategy $C$ is a static storage strategy, that is, pod storage strategy is fixed without any dynamic storage optimization. Table 7 shows the comparison of various simulation strategies under different warehouse sizes, in which the data represents the number of orders completed by a single picking station in a unit hour.

According to the data in table 8 , when the warehouse scale is small, strategy $\mathrm{C}$ is better than strategy $\mathrm{B}$, but the gap between them is small. This is because the eliminated robots in strategy B do not directly participate in order picking under the condition of a certain number of robots, so the performance of strategy $B$ is the worst when the storage scale is small. When the storage scale reaches medium scale, strategy A becomes the best one among the three strategies. When the storage scale is large, strategy B performs best among the three strategies. This is because when the storage scale is large, the overall size of the warehouse is too large, and the moving distance of the pod in the warehouse increases correspondingly during the picking process. The buffer area can effectively cache some high-frequency used pod, which indirectly reduces the moving distance in the picking process, thus increasing the picking efficiency of the picking table.

Table 8 Simulation comparison of different strategies in different scale warehouses

\begin{tabular}{ccccc}
\hline \multirow{2}{*}{$\begin{array}{c}\text { Warehouse } \\
\text { size }\end{array}$} & $\begin{array}{c}\text { Order } \\
\text { quantity }\end{array}$ & $\begin{array}{c}\text { Strategy } \\
\text { A }\end{array}$ & Strategy B & $\begin{array}{c}\text { Strategy } \\
\text { C }\end{array}$ \\
\hline \multirow{2}{*}{ Scenario 1 } & 1500 & 213.97 & 186.67 & 214.64 \\
& 2500 & 211.23 & 175.94 & 210.51 \\
\hline Scenario 2 & 500 & 216.03 & 202.29 & 215.62
\end{tabular}

\begin{tabular}{|c|c|c|c|c|}
\hline & 1500 & 212.73 & 196.16 & 212.06 \\
\hline & 2500 & 212.74 & 195.30 & 210.60 \\
\hline \multirow{3}{*}{ Scenario 3} & 500 & 218.44 & 205.76 & 217.86 \\
\hline & 1500 & 213.32 & 199.53 & 209.47 \\
\hline & 2500 & 210.88 & 197.01 & 209.00 \\
\hline \multirow{3}{*}{ Scenario 4} & 500 & 215.59 & 202.77 & 214.44 \\
\hline & 1500 & 205.24 & 195.06 & 203.20 \\
\hline & 2500 & 204.88 & 195.01 & 203.79 \\
\hline \multirow{3}{*}{ Scenario 5} & 500 & 218.66 & 217.21 & 216.67 \\
\hline & 1500 & 218.91 & 217.08 & 215.83 \\
\hline & 2500 & 217.66 & 216.85 & 214.35 \\
\hline \multirow{3}{*}{ Scenario 6} & 500 & 214.18 & 213.67 & 212.36 \\
\hline & 1500 & 214.86 & 212.87 & 211.93 \\
\hline & 2500 & 213.19 & 212.65 & 212.04 \\
\hline \multirow{3}{*}{ Scenario 7} & 500 & 217.32 & 218.98 & 216.00 \\
\hline & 1500 & 217.11 & 217.30 & 215.92 \\
\hline & 2500 & 217.25 & 217.38 & 215.57 \\
\hline \multirow{3}{*}{ Scenario 8} & 500 & 215.31 & 216.72 & 213.13 \\
\hline & 1500 & 214.57 & 215.59 & 213.44 \\
\hline & 2500 & 214.44 & 215.61 & 213.37 \\
\hline \multirow{3}{*}{ Scenario 9} & 500 & 214.67 & 216.14 & 212.96 \\
\hline & 1500 & 214.14 & 215.18 & 212.71 \\
\hline & 2500 & 214.32 & 215.47 & 212.24 \\
\hline
\end{tabular}

The advantages and disadvantages of storage allocation in the picking process can be reflected through the statistics of the walking path of the handling robot in the picking process. Figure 7 shows the walking thermal diagram of the robot in the medium-sized picking system. The darker the color is, the more frequent the robot visits the position. The red area on the right of the figure is the picking station area. It can be seen from the three groups of thermal diagram that in this scenario, the most intensive storage areas accessed by strategy A robots are concentrated in the best-selling areas and some areas with general sales volume; the most intensive storage areas accessed by strategy B robot are concentrated in the buffer area and some areas close to the picking table; In strategy $\mathrm{C}$, the robot access to the thermal diagram is relatively uniform, and the 
centralized area of thermal diagram covers most of the storage area. In the thermal diagram of this scenario, the centralized area in strategy A is closest to the picking platform, which reflects that the average moving distance of the robot in the picking process is short, while the average moving distance in strategy $\mathrm{C}$ is the longest.

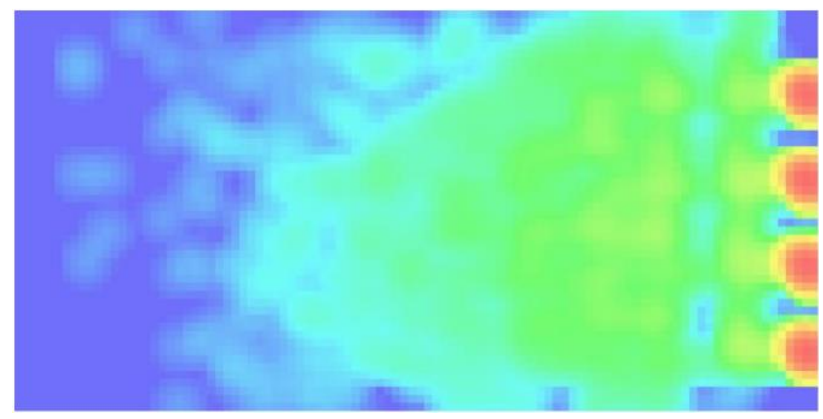

(a) Strategy A

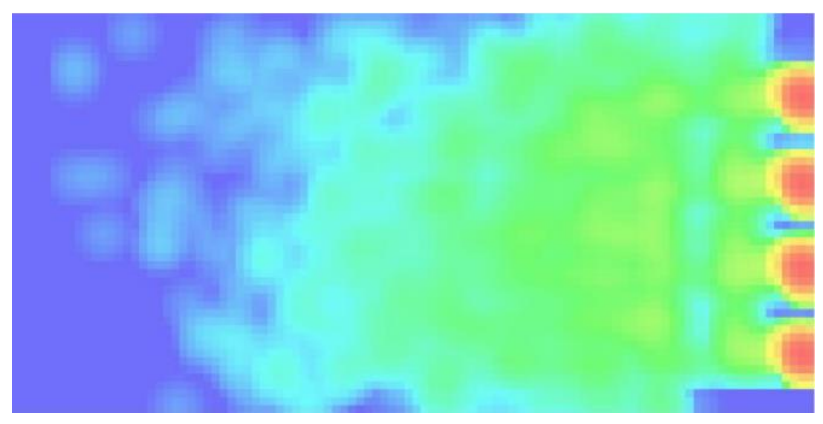

(b) Strategy B

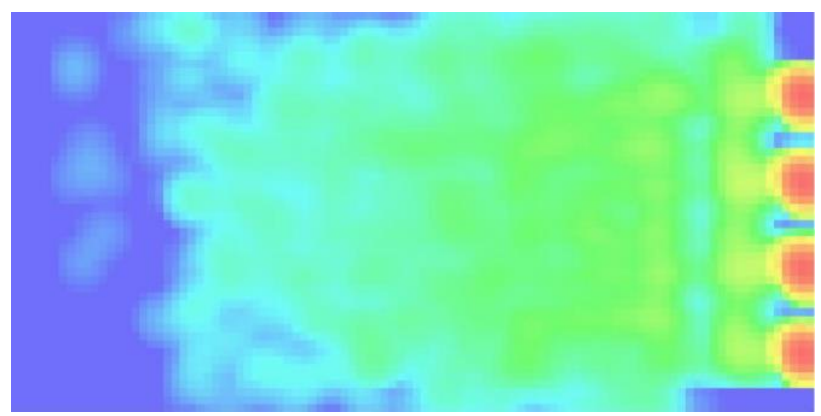

(c) Strategy C

Figure 7 Thermodynamic diagram of robot walking in the medium scale picking system

Figure 8 shows the walking thermodynamic diagram of the robot in the picking process of the large-scale picking system. Due to the large storage scale in this scenario, the buffer area of strategy B can effectively reduce the moving distance of the robot, so the most concentrated walking path in figure (b) is concentrated in the buffer area, and the concentrated area of strategy B is closer to the picking platform than that of strategy a, while the average moving distance of the robot in the picking process of strategy $\mathrm{C}$ is longer due to the fixed storage location.

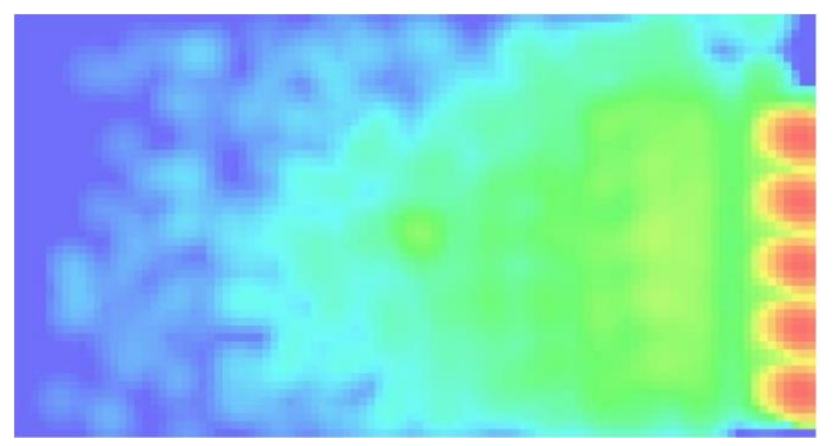

(a) Strategy A

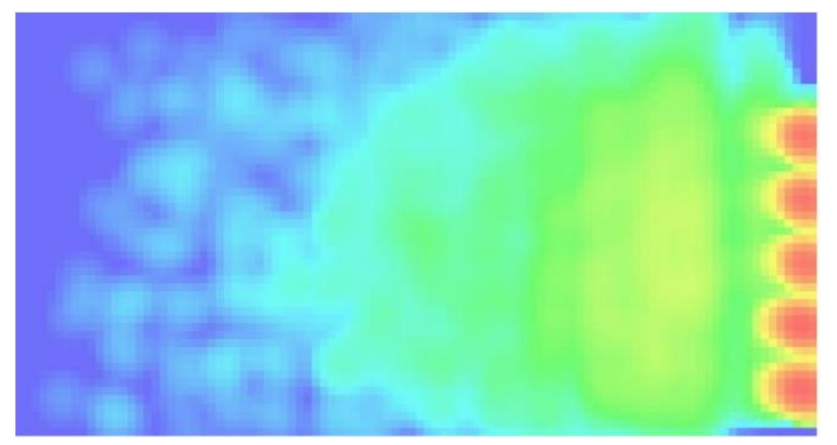

(b) Strategy B

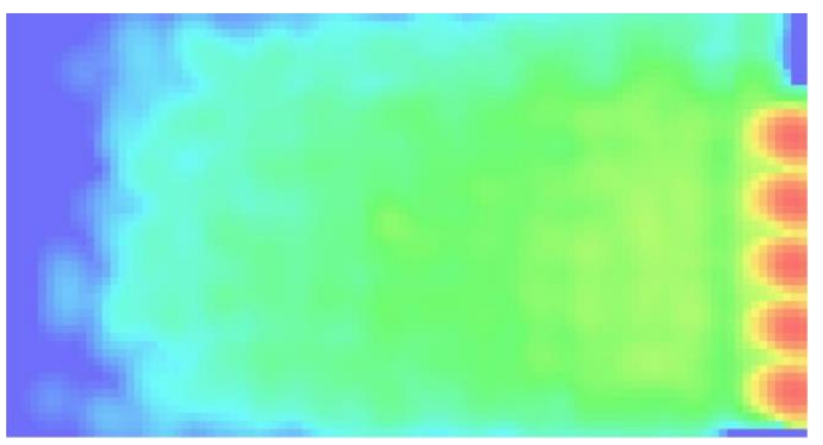

(c) Strategy C

Figure 8 Thermodynamic diagram of robot walking in the large scale picking system

\section{CONCLUSION}

This paper first introduces the related concepts of dynamic storage allocation, and takes the pod handling robot picking system as an example to analyze the problems to be solved. Then, a dynamic storage allocation model suitable for the pod handling robot picking system is constructed, and two dynamic storage allocation strategies are proposed for solving the model-partition dynamic storage strategy and buffer based dynamic storage strategy. 
According to the different modes, the partition dynamic storage strategy is divided into the partition strategy based on the pod turnover rate and the partition strategy based on the item turnover rate. The buffer based storage strategy is divided into the first in first out elimination strategy, the elimination strategy according to the frequency of use, the least frequently used elimination strategy and the random elimination strategy, and the characteristics of each strategy are introduced in detail. Finally, simulation experiments are carried out on different scale picking systems to find out the optimal configuration of the partition storage strategy and cache storage strategy to adapt to different systems. Finally, by comparing the static storage strategy, the effectiveness of the dynamic storage strategy is proved. The partition storage strategy is more suitable for the small and medium-sized warehouse system, and the buffer storage strategy performs better than the large-scale picking system.
[10]De Koster R, Le-Duc T, Roodbergen K J. Design and control of warehouse order picking: A literature review[J]. European Journal of Operational Research, 2007,182(2):481-501.

[11]Wurman, Peter, R. Coordinating Hundreds of Cooperative, Autonomous Vehicles in Warehouses.[J]. Ai Magazine, 2008.

[12]Lamballais T, Roy D, De Koster M B M. Inventory Allocation in Robotic Mobile Fulfillment Systems[J]. Social Science Electronic Publishing, 2017.

[13]Duguleana M, Mogan G. Neural networks based reinforcement learning for mobile robots obstacle avoidance[J]. Expert Systems with Applications, 2016,62:104-115.

[14]Lee C K M, Lin B, Ng K K H, et al. Smart robotic mobile fulfillment system with dynamic conflict-free strategies considering cyberphysical integration[J]. Advanced Engineering Informatics, 2019,42.

[15]Merschformann M X L L. RAWSim-O: A Simulation Framework for Robotic Mobile Fulfillment Systems[J]. Logistics Research, 2018, 11(1).

\section{DECLARATION}

\section{Acknowledgements}

The authors sincerely thanks to Professor $\mathrm{Wu}$ of Shandong University for his critical discussion and reading during manuscript preparation.

\section{Funding}

Not applicable

\section{References}

[1] BARTHOLDI III J J, HACKMAN S T, Warehouse and distribution science[M]. Atlanta: Georgia Institute of Technology, 2008.

[2] Wurman, Peter, R. Coordinating Hundreds of Cooperative, Autonomous Vehicles in Warehouses.[J]. Ai Magazine, 2008.

[3] Mountz MC, D'Andrea R, Laplante J A, et al. Inventory system with mobile drive unit and inventory holder: US 2008.

[4] Bipan Zou, Yeming (Yale) Gong, Xianhao Xu and Zhe Yuan. Assignment rules in robotic mobile fulfilment systems for online retailers. International Journal of Production Research, 2017, Vol. 55, No. 20, 6175-6192.

[5] Nigam S, Roy D, De Koster R, Adan I (2014) Analysis of class-based storage strategies for the mobile shelf-based order pick system. Smith J, Ellis K, De Koster R, Lavender S, Montreuil B, Ogle M, eds. 13th IMHRC Proc. (CICMHE, Charlotte, NC), 19.

[6] Yuan R, Cezik T, Graves S C. Velocity-Based Storage Assignment in Semi-Automated Storage Systems[J]. SSRN Electronic Journal, 2016.

[7] Lamballais, T, Roy, D, De Koster, M.B.M. Estimating performance in a Robotic Mobile Fulfillment System[J]. European Journal of Operational Research, 2017, 256(3):976-990.

[8] Boysen N, Briskorn D, Emde S. Parts-to-picker based order processing in a rack-moving mobile robots environment[J]. European Journal of Operational Research, 2017:550-562.

[9] Bipan Zou, Xianhao Xu , Yeming (Yale) Gong, René De Koster. Evaluating battery charging and swapping strategies in a robotic mobile fulfillment system. European Journal of Operational Research, Volume 267, Issue 2, 1 June 2018: 733-753

\section{Availability of data and materials}

The datasets supporting the conclusions of this article are included within the article.

\section{Authors' contributions}

The author' contributions are as follows: Xia and Chi was in charge of the trial; Chi wrote the manuscript; Wu assisted with sampling and laboratory analyses.

\section{Competing interests}

The authors declare no competing financial interests.

\section{Consent for publication \\ Not applicable}

\section{Ethics approval and consent to participate}

Not applicable 